Pacific Journal of Mathematics

COMPACT CONNECTED LIE GROUPS ACTING ON SIMPLY

HAE So O 


\title{
COMPACT CONNECTED LIE GROUPS ACTING ON SIMPLY CONNECTED 4-MANIFOLDS
}

\author{
HaE SOO OH
}

\begin{abstract}
Suppose a compact connected Lie group $G$ acts effectively on a simply connected 4-manifold $M$. Then we show that $G$ is one of the groups $\mathrm{SO}(5), \mathrm{SU}(3) / Z(G), \mathrm{SO}(3) \times \mathrm{SO}(3), \mathrm{SO}(4), \mathrm{SO}(3) \times T^{1}$, $\left(\mathrm{SU}(2) \times T^{1}\right) / D, \mathrm{SU}(2), \mathrm{SO}(3), T^{2}, T^{1}$, and that the representatives of the conjugacy classes of the principal isotropy groups for these groups on $M$ are, respectively, $\mathrm{SO}(4), U(2), T^{2}, \mathrm{SO}(3), S^{1}, S^{1}, \widetilde{\mathrm{SO}}(2)$ or $e, \mathrm{SO}(2)$ or $D_{2 n}, e$, and $e$. We also show that in each of these cases $M$ is a connected sum of copies of $S^{4}, S^{2} \times S^{2}, C P^{2}$, and $-C P^{2}$ (except when $G$ is $T^{1}$, see Theorem 2.6).
\end{abstract}

1. Introduction. All manifolds in this paper are assumed to be closed, connected and orientable. Also all actions are assumed to be effective and locally smooth. Orlik-Raymond [O-R] showed that if a simply connected 4-manifold admits an action of the two-dimensional torus group $T^{2}$, then $M$ is a connected sum of copies of $S^{4}, S^{2} \times S^{2}$, $C P^{2}$, and $-C P^{2}$. Fintushel $\left[\mathbf{F}_{2}\right]$ proved that if $M$ admits a circle action and the orbit space $M^{*}$ is not a counterexample of Poincarés conjecture, then $M$ is also a connected sum of copies of these manifolds.

In this paper we determine all Lie groups which can act on a simply connected 4-manifold $M$, and dually we classify all simply connected 4-manifolds which admit an action of a given compact connected Lie group $G$.

An isotropy group $H$ is a principal if $H$ is conjugate to a subgroup of each isotropy group (that is, $G / H$ is a maximum orbit type for $G$ on $M$ ). One denotes by $G(x)$ the orbit of $G$ through $x$, and by $G_{x}$ the isotropy group at $x$. A maximal torus $T$ is a compact connected abelian Lie subgroup which is not properly contained in any larger such subgroup. We denote the normalizer of $G$ by $N(G)$, and the centralizer of $G$ by $Z(G)$. Let $\chi(M)$ denote the euler characteristic of a space $M$. Then it is well known that $\chi(G / T)$ is the order of $N(T) / T$.

2. The rank of a Lie group $G$ which can act on a simply connected 4-manifold $M$. Suppose $K$ is a subgroup of $G$ which acts on a topological space $X$. Then the action of $G$ on $X$ may not be effective even if the action restricted to $K$ is effective. But the maximal torus theorem gives rise to the following. 
LeMma 2.1. A compact connected Lie group $G$ acts effectively on a topological space $X$ if and only if the action restricted to a maximal torus $T$ of $G$ is effective.

Proof. Suppose $G$ does not act effectively. Then there exists at least one element $g \neq e$ in $G$ such that $g x=x$, for all $x \in X$. It follows from the maximal torus theorem that there exists an element $h \in G$ such that $g \in h T h^{-1}$. Hence $h^{-1} g h \in T$. Thus we have $\left(h^{-1} g h\right) x=h^{-1} g(h x)=$ $h^{-1} h x=x$, for all $x \in X$, which says that the action restricted to $T$ is not effective.

By the rank of a Lie group $G$, we mean the dimension of a maximal torus of $G$.

LEMMA 2.2. If a compact connected Lie group $G$ acts on a simply connected 4-manifold $M$, then the rank of $G$ is less than 3.

Proof. Suppose the rank of $G$ is $\geq 3$. Then $M$ admits an effective $T^{3}$-action. By [P], $M$ is homeomorphic to either $T^{4}$ or $L(p, q) \times T^{1}$, which contradicts the simple connectivity of $M$.

It is known that every compact connected Lie group of dimension $\leq 6$ can be represented as a factor group $G / F$, where $G=G_{1} \times G_{2}$ $\times \cdots \times G_{n}$ is a product; each factor $G_{i}$ is either $\mathrm{SO}(2)$ or $\mathrm{SU}(2)\left(=S^{3}\right)$, and $F$ is a finite subgroup of the center of $G$.

From now on $G$ is a compact connected Lie group acting on a simply connected 4-manifold $M$, and $H$ is a principal isotropy group for $G$ on $M$. (Note: any two principal isotropy groups are conjugate to each other. Actually $H$ denotes a representative group of the conjugacy class of principal isotropy groups.)

LEMMA 2.3. Suppose the rank of $G$ is 2 and the rank of $H$ is 0 . Then $G$ is the two-dimensional torus group $T^{2}$.

Proof. From [B, p. 195], we have the following inequality:

(*) $\operatorname{dim} M-\operatorname{dim} G / H-(\operatorname{rank} G-\operatorname{rank} H) \leq \operatorname{dim} M-2 \operatorname{rank} G$.

Since we assumed $\operatorname{rank} H=0$, then $\operatorname{dim} G / H \leq 4$. Hence the inequality gives rise to $4 \geq \operatorname{dim} G / H \geq \operatorname{rank} G=2$. Since $\operatorname{dim} G-\operatorname{rank} G$ should be an even integer, $\operatorname{dim} G(=\operatorname{dim} G / H)$ must be either 4 or 2 . 
If $\operatorname{dim} G$ is 4 , then $G$ acts transtively on $M$ (that is, $M=G / H$ ). Since a compact connected Lie group of dimension 4 and of rank 2 is either $\mathrm{SU}(2) \times \mathrm{SO}(2)$ or a factor group of this by a finite subgroup, $G / H$ cannot be simply connected. We thus have $\operatorname{dim} G=2=\operatorname{rank} G$. Hence $G$ is $T^{2}$.

LEMMA 2.4. If a compact connected Lie group $G$ acts on a simply connected 4-manifold $M$, then we have the following:

(i) if rank $G=2$ and rank $H=2$, then $\operatorname{dim} G$ is 10,8 , or 6;

(ii) if $\operatorname{rank} G=2$ and $\operatorname{rank} H=1$, then $\operatorname{dim} G / H=3$ and $\operatorname{dim} G$ is either 6 or 4 ;

(iii) if rank $G=2$ and rank $H=0$, then $G=T^{2}$;

(iv) the orbit space $M^{*}$ is a simply connected manifold with boundary.

Proof. From [B, p. 195], we have an inequality,

$$
4 \geq \operatorname{dim} G / H \geq \operatorname{rank} G+\operatorname{rank} H .
$$

It is known [M-Z] that if the maximal dimension of any orbit is $k$, then $\operatorname{dim} G \leq k(k+1) / 2$. Thus $\operatorname{dim} G \leq 10$. Since $\operatorname{dim} G-\operatorname{rank} G$ is an even integer, $\operatorname{dim} G$ is $10,8,6,4$, or 2 , provided rank $G$ is 2 .

(i) If $\operatorname{rank} G=2$ and $\operatorname{rank} H=2$, then it follows from inequality (**) that $\operatorname{dim} G / H=4$. Hence $\operatorname{dim} G \geq 6$.

(ii) If $\operatorname{rank} G=2$ and $\operatorname{rank} H=1$, then by $(* *), \operatorname{dim} G / H$ is either 3 or 4. Suppose $\operatorname{dim} G / H=4$. Then $\operatorname{dim} H(=\operatorname{dim} G-\operatorname{dim} G / H)$ is 6,4 , or 2. On the other hand, rank $H=1$ implies that the identity component of $H$ is $\mathrm{SO}(2), \mathrm{SO}(3)$, or $\mathrm{SU}(2)$. Hence $\operatorname{dim} G / H$ should be 3 . By [M-Z], $\operatorname{dim} G \leq \frac{1}{2}(\operatorname{dim} G / H)(\operatorname{dim} G / H+1)=6$.

(iii) was shown in Lemma 2.3.

(iv) If rank $G=2$ and $\operatorname{rank} H \geq 1$, then (**) implies that $\operatorname{dim} G / H$ is either 4 or 3. If $\operatorname{rank} G=2$, and $\operatorname{rank} H=0$, then by (iii), we have $G=T^{2}$.

Thus if rank $G=2$, the orbit space $M^{*}$ is $D^{0}, D^{1}$, or $D^{2}$ (cf. Lemma 5.1 [O-R]). If rank $G=1$, then $G$ is $\mathrm{SO}(2), \operatorname{SO}(3)$, or $\mathrm{SU}(2)$. Since any proper subgroups of $\mathrm{SO}(3)$ and $\mathrm{SU}(2)$ are of dimension $\leq 1$, if $G$ is either $\mathrm{SO}(3)$ or $\mathrm{SU}(2)$, then $\operatorname{dim} G / H$ should be $\geq 2$. Hence $M^{*}$ is $D^{1}, D^{2}$, or $S^{2}$. If $G=\mathrm{SO}(2)$, then by Lemma $3.1\left[\mathbf{F}_{1}\right], M^{*}$ is a simply connected 3-manifold with boundary.

If an abelian group $G$ acts effectively on a manifold $M$, then the principal isotropy group $H$ is trivial. We have shown that if $\operatorname{rank} G=2$ 
and $\operatorname{rank} H=0$, then $G$ is $T^{2}$, hence $H$ is trivial. In this case, the manifolds are determined by the following theorem.

THEOREM 2.5. [O-R] If $M$ is a simply connected 4-manifold supporting an effective $T^{2}$-action, then $M$ has $k(\geq 2)$-fixed points, and

$$
M \approx\left\{\begin{array}{l}
S^{4}, \quad \text { if } k=2 ; \\
C P^{2} \text { or }-C P^{2}, \quad \text { if } k=3 \\
S^{2} \times S^{2}, C P^{2} \# C P^{2}, C P^{2} \#-C P^{2}, \text { or }-C P^{2} \#-C P^{2}, \quad \text { if } k=4 \\
\text { a connected sum of copies of these spaces, if } k>4 .
\end{array}\right.
$$

THEOREM 2.6. $\left[\mathbf{F}_{\mathbf{2}}\right]$ Let $\mathrm{SO}(2)$ act locally smoothly and effectively on the simply connected 4-manifold $M$, and suppose the orbit space $M^{*}$ is not a counterexample to the 3-dimensional Poincaré conjecture. Then $M$ is a connected sum of copies of $S^{4}, C P^{2},-C P^{2}$, and $S^{2} \times S^{2}$.

Suppose a compact Lie group $G$ acts on a compact connected manifold $M$ so that the orbit space $M / G$ is a closed interval $[0,1]$, and let $G(x)$ and $G(y)$ be the orbits corresponding to 0 and 1 respectively. Then $G(x)$ and $G(y)$ are singular orbits and all other orbits are principal orbits of type $G / H$. Moreover, we may assume $H \subset G_{x}$ and $H \subset G_{v}$. The following lemma was proved by Mostert [Mo].

LEMMA 2.7. [Mo] If a Lie group $G$ acts locally smoothly and effectively on a manifold $M$ so that $M / G$ is a closed interval, then $G_{\downarrow} / H$ and $G_{1} / H$ are spheres.

3. The case of rank $G=2$.

3A. Suppose rank $G=2$ and rank $H=2$. Then by Lemma $2.4 \operatorname{dim} G$ is 10,8 , or 6 . Inequality $(* *)$ implies $\operatorname{dim} G / H=4$ and hence $M$ is a homogeneous space.

(i) It follows from [E, p. 239] that if $\operatorname{dim} G=10$, then $M$ is $S^{4}$ or $R P^{4}$. Since $M$ is simply connected, $M$ is $S^{4}$. Hence [Wo, p. 282] gives rise to $G=\mathrm{SO}(5)$ and $H=\mathrm{SO}(4)$.

(ii) It is known [Wa] that if $n(n-1) / 2+1<\operatorname{dim} G<n(n+1) / 2$, $n=\operatorname{dim} M$, then $n=4$. Mann [Ma] proved that the effective action of $\mathrm{SU}(3) / Z(\mathrm{SU}(3))$ of dimension 8 on the complex projective plane $C P^{2}=$ $\mathrm{SU}(3) / U(2)$ is the only exceptional possibility for $n=4$.

(iii) If $\operatorname{dim} G=6$, then $\operatorname{dim} H$ should be 2 . Since $G$ is assumed to be connected and $G / H=M$ is assumed to be simply connected, the homotopy exact sequence of the fibre bundle implies that $H$ is also connected, 
hence $H$ is $T^{2}$. The Lie group $G$ of dimension 6 and of rank 2 is either $\mathrm{SU}(2) \times \mathrm{SU}(2)$, or a factor group of this by a finite subgroup. Since $Z(\operatorname{SU}(2) \times \mathrm{SU}(2))=\{(1,1),(-1,1),(1,-1),(-1,-1)\}$ is contained in a maximal torus (and hence in $H$ ), $\mathrm{SU}(2) \times \mathrm{SU}(2)$ is not admissible. For similar reasons, $\mathrm{SO}(3) \times \mathrm{SU}(2), \mathrm{SU}(2) \times \mathrm{SO}(3)$, and $\mathrm{SO}(4)$ are not admissible. Hence $(\mathrm{SU}(2) \times \mathrm{SU}(2)) /$ the center $=\mathrm{SO}(3) \times \mathrm{SO}(3)$ is the only admissible group. Hence $M$ is $S^{2} \times S^{2}$.

We recall some properties of $\mathrm{SO}(3)$ (see $[\mathbf{R}]$ ).

(1) Every subgroup of $\mathrm{SO}(3)$ is conjugate to one of the following: $\mathrm{SO}(2), N(\mathrm{SO}(2))$, the cyclic group $Z_{k}$ of order $k$, the dihedral group $D_{n}$ of order $2 n$, the groups $T, O, I$ of all rotational symmetries of the tetrahedron, octahedron, and icosahedron, respectively.

(2) If $V$ is a finite subgroup of $\mathrm{SO}(3)$, then $\mathrm{SO}(3) / V$ is an orientable 3-manifold with $\mathrm{H}_{2}(\mathrm{SO}(3) / V)=0$. Using the double covering $\pi: \mathrm{SU}(2)$ $\rightarrow \mathrm{SO}(3)$ we can calculate the first homology group of $\mathrm{SO}(3) / V$ :

$$
\begin{array}{ll}
H_{1}\left(\mathrm{SO}(3) / Z_{k}\right)=Z_{2 k}, & H_{1}\left(\mathrm{SO}(3) / D_{2 n}\right)=Z_{2}+Z_{2}, \\
H_{1}\left(\mathrm{SO}(3) / D_{2 n+1}\right)=Z_{4}, & H_{1}(\mathrm{SO}(3) / T)=Z_{3}, \\
H_{1}(\mathrm{SO}(3) / O)=Z_{2}, & H_{1}(\mathrm{SO}(3) / I)=0
\end{array}
$$

In the following $\tilde{K}$ denotes the preimage of $K \subset \mathrm{SO}(3)$ under the covering map.

3B. Suppose $\operatorname{rank} G=2$ and $\operatorname{rank} H=1$. Then by Lemma $2.4, \operatorname{dim} G$ is either 6 or 4 and $\operatorname{dim} G / H$ is 3 .

(I) If $\operatorname{dim} G=4$, then $G$ is $\mathrm{SU}(2) \times T^{1}$ or a factor group of this by a finite subgroup. Since $\operatorname{dim} G / H$ is $3, \operatorname{dim} H$ is 1 . Since any 1-dimensional subgroup of $\mathrm{SU}(2) \times T^{1}$ contains a non-trivial element of $\mathrm{Z}\left(\mathrm{SU}(2) \times T^{1}\right)$ $=\{1,-1\} \times T^{1}$, it is not admissible. The remaining possibilities are $\mathrm{SO}(3) \times T^{1}$ and $\left(\mathrm{SU}(2) \times T^{1}\right) / D$, where $D=\{(1,1),(-1,-1)\}$.

(Ia) Suppose $G$ is $\left(\mathrm{SU}(2) \times T^{1}\right) / D$. Then the identity component $H_{0}$ of $H$ cannot be included in $(\mathrm{SU}(2) \times 1) / D$ since $(\widetilde{\mathrm{SO}}(2) \times 1) / D$ contains $(-1,1) / D(\in Z(G))$. Nor can $H_{0}$ be $\left(1 \times T^{1}\right) / D$ since $\left(1 \times T^{1}\right) / D$ is a subgroup of $Z(G)$. Hence by using an argument similar to that of 8.1 of [R], we can show that $H$ is included in a maximal torus of $G$.

Since $\operatorname{dim} G / H$ is 3 , the orbit space $M^{*}$ is a closed interval $[0,1]$. That is, the orbit space $M^{*}$ is as shown below.

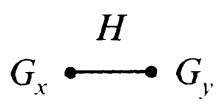

By Lemma 2.7, $G_{x} / H$ and $G_{y} / H$ are spheres. But $\left(\left(\widetilde{\mathrm{NSO}}(2) \times T^{1}\right) / D\right) / H$ is not a sphere. Hence $G_{x}$ (and also $G_{y}$ ) must be $\left(\widetilde{\mathrm{SO}}(2) \times T^{1}\right) / D$ or $G$. 
(i) If $G_{x}$ and $G_{v}$ are maximal tori, then the number of fixed points of the action restricted to $G_{x}$ is either 2 or 4 since the order of $N\left(G_{x}\right) / G_{x}$ is $\chi\left(G / G_{x}\right)=2$. Now it follows from Theorem 2.5 that $M$ is $S^{4}$ or an $S^{2}$-bundle over $S^{2}$ according as the number of fixed points is 2 or 4 . Let $A=p^{-1}\left(\left[0, \frac{1}{2}\right]\right)$ and $B=p^{-1}\left(\left[0, \frac{1}{2}\right]\right)$, where $p: M \rightarrow M^{*}=[0,1]$ is the orbit map. From the Mayer-Vietoris sequence for $(M, A, B)$, we have

$$
0 \rightarrow H_{3}(M) \rightarrow H_{2}(G / H) \rightarrow Z \oplus Z \rightarrow H_{2}(M) \rightarrow H_{1}(G / H) \rightarrow 0 .
$$

Now we have

$$
\begin{aligned}
(G / H) /\left\{\left[\left(\widetilde{\mathrm{SO}}(2) \times T^{1}\right) / D\right] / H\right\} \\
\quad \approx\left[\left(\mathrm{SU}(2) \times T^{1}\right) / D\right] /\left[\left(\widetilde{\mathrm{SO}}(2) \times T^{1}\right) / D\right] \\
\approx\left(\mathrm{SU}(2) \times T^{1}\right) /\left(\widetilde{\mathrm{SO}}(2) \times T^{1}\right) \approx S^{2}
\end{aligned}
$$

(see [B, p. 87]). Since $\left[\left(\widetilde{\mathrm{SO}}(2) \times T^{1}\right) / D\right] / H$ is a topological group, the fundamental group of this is abelian. From a homotopy exact sequence of the fibre bundle $\left[\left(\widetilde{\mathrm{SO}}(2) \times T^{1}\right) / D\right] / H \rightarrow G / H \rightarrow S^{2}$, we can see that $\pi_{1}(G / H)$ is abelian, hence $H_{1}(G / H)=\pi_{1}(G / H)$.

From the homotopy sequence of the fibre bundle $H \rightarrow G \rightarrow G / H$, we have

$$
0 \rightarrow \pi_{2}(G / H) \rightarrow Z \rightarrow Z \rightarrow \pi_{1}(G / H) \rightarrow \pi_{0}(H) \rightarrow 0 .
$$

If $M$ is $S^{4}$, then from the homology sequence we have $\pi_{2}(G / H)=Z \oplus Z$ which contradicts the homotopy sequence. Hence the number of fixed points must be 4 . Therefore we have $G_{x}=G_{v}$ which implies $\pi_{1}(G / H)=1$, and hence $H$ is connected. Thus $H$ is $S^{1}$ and $M$ is either $S^{2} \times S^{2}$ or $C P^{2} \#-C P^{2}$.

(ii) If $G_{x}$ and $G_{v}$ are $G$ (i.e. $x$ and $y$ are fixed points), then the homotopy exact sequence of a fibre bundle $H \rightarrow G \rightarrow G / H=G_{x} / H \approx S^{3}$ yields $H \approx S^{1}$. Furthermore, the number of fixed points of the action restricted to $\left(\widetilde{\mathrm{SO}}(2) \times T^{1}\right) / D$ is two. Hence, by Theorem 2.5 , we have $M=S^{4}$ (alternatively,

$$
\left.M \approx p^{-1}\left(\left[0, \frac{1}{2}\right]\right) \cup p^{-1}\left(\left[\frac{1}{2}, 1\right]\right) \approx D^{4} \cup D^{4} \approx S^{4}\right) .
$$

(iii) If $G_{x}$ is $G$ and $G_{y}$ is a maximal torus, then by an argument similar to that used in (ii), $H$ is connected and hence $H$ is $S^{1}$. The number of fixed points of the action restricted to $G_{y}$ is 3 and hence it follows from Theorem 2.5 that $M$ is $C P^{2}$.

(Ib) Suppose $G$ is $\operatorname{SO}(3) \times T^{1}$. Then by 8.1 of $[\mathbf{R}], H$ is contained in a maximal torus or conjugate to either $\mathrm{SO}(2) \times 1$ or $N(\operatorname{SO}(2)) \times 1$. But 
$(\mathrm{SO}(3) \times 1) /(N(\operatorname{SO}(2)) \times 1)=R P^{2} \times S^{1}$ is not orientable and hence by [B, p. 188], $H$ cannot be $N(\mathrm{SO}(2)) \times 1$. (1) If $H$ is contained in a maximal torus, then neither $G_{x}$ nor $G_{y}$ can be $G$ since $\left(\operatorname{SO}(3) \times T^{1}\right) / H$ is not a sphere. Hence by an argument similar to that of (Ia), $H$ is $S^{1}$ and $M$ is $S^{2} \times S^{2}$ or $C P^{2} \#-C P^{2}$. (2) If $H$ is $\mathrm{SO}(2) \times 1$, then by Lemma 2.7 , there are three possibilities:

(i) $G_{x} \approx \mathrm{SO}(2) \times T^{1} \approx G_{y}$, which implies $M=S^{2} \times S^{2}$.

(ii) $G_{x} \approx \mathrm{SO}(3)$ and $G_{y} \approx \mathrm{SO}(2) \times T^{1}$, which implies $M=\left[\left(S^{2} \times D^{2}\right)\right.$ $\left.\cup\left(D^{3} \times S^{1}\right)\right]=S^{4}$.

(iii) $G_{x} \approx \mathrm{SO}(3) \approx G_{v}$, which implies $M=S^{3} \times S^{1}$, not admissible.

(II) If $\operatorname{dim} G=6$, then $\operatorname{dim} H$ should be 3 . Since the rank of $G$ is $2, G$ is $\mathrm{SU}(2) \times \mathrm{SU}(2), \mathrm{SO}(3) \times \mathrm{SU}(2), \mathrm{SU}(2) \times \mathrm{SO}(3)$, or $(\mathrm{SU}(2) \times \mathrm{SU}(2)) / D$, where $D=\{(1,1),(-1,-1)\}$.

Assertion. Suppose $H_{0}$ is the identity component of a 3-dimensional subgroup $H$ of $\mathrm{SU}(2) \times \mathrm{SU}(2)$ and let $p_{i}$ be the projection onto the $i$ th factor, for $i=1,2$. Then $p_{i} \mid H_{0}$, the restriction of $p_{1}$ to $H_{0}$, is either a trivial map or an isomorphism.

To prove this Assertion, first of all we have to show that $p_{i} \mid H_{0}$ is either trivial or surjective. Suppose $p_{l} \mid H_{0}$ is neither surjective nor trivial. Then $p_{i}\left(H_{0}\right)$ should be either $\mathrm{SO}(2)$ or $N(\mathrm{SO}(2))$, and hence the kernel of $p_{\imath} \mid H_{0}$ is a two-dimensional normal subgroup of $H_{0}$. This is impossible. Hence $p_{1} \mid H_{0}$ or $p_{2} \mid H_{0}$ must be surjective. Suppose $p_{1} \mid H_{0}$ is surjective and let $K$ be the kernel of $p_{1} \mid H_{0}$. Then $H_{0} / K \approx \mathrm{SU}(2)$. Since $\mathrm{SO}(3)$ is simple, $H_{0}$ cannot be $\mathrm{SO}(3)$. If $H_{0}$ is $\mathrm{SU}(2)$, then $K=\pi_{1}\left(H_{0} / K\right)=\pi_{1}(\mathrm{SU}(2))=1$. Thus $p_{1} \mid H_{0}$ is an isomorphism and $H_{0} \approx \mathrm{SU}(2)$.

(IIa) If either $p_{1} \mid H_{0}$ or $p_{2} \mid H_{0}$ is trivial, then $H \approx \mathrm{SU}(2) \times V$, for a finite subgroup $V$, which contains a normal subgroup of $\mathrm{SU}(2) \times \mathrm{SU}(2)$. Since $H$ cannot contain a normal subgroup of $\mathrm{SU}(2) \times \mathrm{SU}(2), p_{1} \mid H_{0}$ and $p_{2} \mid H_{0}$ must be isomorphisms. Therefore, $H$ contains the two elements central subgroup $D$. Thus $\mathrm{SU}(2) \times \mathrm{SU}(2)$ is not admissible.

(IIb) If $G$ is $\mathrm{SO}(4)(\approx(\mathrm{SU}(2) \times \mathrm{SU}(2)) / D)$, then a principal isotropy group is $H / D$, where $H$ is a three-dimensional subgroup of $\mathrm{SU}(2) \times \mathrm{SU}(2)$ such that $p_{1}(H)=\mathrm{SU}(2)=p_{2}(H)$.

If $x^{*}$ and $y^{*}$ are the endpoints of a closed interval $M / \mathrm{SO}(4)$, then $x$ and $y$ should be fixed points so that $G_{x}$ (and also $G_{y}$ ) could contain $H$ as a conjecture subgroup. In fact, suppose $K$ is a subgroup of $\mathrm{SU}(2) \times \mathrm{SU}(2)$ such that $H \subset K$ and $\operatorname{dim} K \geq 4$. Then $\operatorname{dim} K$ is either 4 or 6 since rank $G$ is 2 . If $\operatorname{dim} K$ is 4 , then the kernel of $P_{1}$ is an 1-dimensional subgroup of $K$. So $K$ contains $1 \times \widetilde{\mathrm{SO}}(2)$. For any $g \in \mathrm{SU}(2)$, there exists $h \in \mathrm{SU}(2)$ 
such that $(h, g) \in K$. Moreover, $(h, g)^{-1}(1 \times \widetilde{\mathrm{SO}}(2))(h, g)=1 \times$ $g^{-1} \widetilde{\mathrm{SO}}(2) g \subset K$. By the maximal torus theorem, we have $1 \times \mathrm{SU}(2) \subset K$. Similarly, $\mathrm{SU}(2) \times 1 \subset K$. Hence $K=\mathrm{SU}(2) \times \mathrm{SU}(2)$. Since $G /(H / D)$ must be $S^{3}$ (by Theorem 2.7), by a homotopy exact sequence of $H / D \rightarrow G$ $\rightarrow S^{3}, H / D$ is connected. Since $H_{0} / D \approx \mathrm{SU}(2) / D \approx \mathrm{SO}(3), H / D$ is $\mathrm{SO}(3)$ and hence $M$ is $S^{4}$.

(IIc) If $G$ is $\mathrm{SO}(3) \times \mathrm{SO}(3)$, then by an argument similar to the Assertion, we can show that $x$ and $y$ should be fixed points so that $G_{x}$ (and $G_{v}$ ) can contain a non-normal 3-dimensional subgroup $H$ as a conjugate subgroup. But $(\mathrm{SO}(3) \times \mathrm{SO}(3)) / H$ cannot be a sphere. Hence $\mathrm{SO}(3) \times$ $\mathrm{SO}(3)$ is not admissible.

(IId) If $G$ is $\mathrm{SU}(2) \times \mathrm{SO}(3)$, then by an argument similar to that used in the proof of the Assertion, $P_{1} \mid H_{0}$ is either a trivial map or an isomorphism. If $P_{1} \mid H_{0}$ is trivial, then $H$ is $V \times \mathrm{SO}(3)$ for a finite subgroup $V$ of $\mathrm{SU}(2)$, which contains a normal subgroup $1 \times \mathrm{SO}(3)$. If $P_{1} \mid H_{0}$ is an isomorphism, then $H$ contains $\{(-1,1),(1,1)\}(\subset Z(G))$. Hence $\mathrm{SU}(2) \times \mathrm{SO}(3)$ is not admissible.

As a summary we have the table:

TABLE I

\begin{tabular}{|c|c|c|c|l|}
\hline $\operatorname{dim} G$ & rank $H$ & $G$ & $H$ & $M$ \\
\hline 10 & 2 & $\mathrm{SO}(5)$ & $\mathrm{SO}(4)$ & $S^{4}$ \\
8 & 2 & $\mathrm{SU}(3) / Z(G)$ & $U(2) / Z(G)$ & $C P^{2}$ \\
6 & 2 & $\mathrm{SO}(3) \times \mathrm{SO}(3)$ & $T^{2}$ & $S^{2} \times S^{2}$ \\
\hline 6 & 1 & $\mathrm{SO}(4)$ & $\mathrm{SO}(3)$ & $S^{4}$ \\
4 & 1 & $\mathrm{SO}(3) \times T^{1}$ & $S^{1}$ & $S^{2} \times S^{2}, S^{4}, C P^{2} \#-C P^{2}$ \\
4 & 1 & $\mathrm{SU}(2) \times T^{1} / D$ & $S^{1}$ & $S^{4}, C P^{2}, S^{2} \times S^{2}, C P^{2} \#-C P^{2}$ \\
\hline 2 & 0 & $T^{2}$ & $e$ & Theorem 2.5 \\
\hline
\end{tabular}

Here $S^{1}$ is a circle subgroup and $D$ is the two element central subgroup $\{(1,1),(-1,-1)\}$.

4. The case of rank $G=1$. If a compact connected Lie group $G$ is of rank 1 , then $G$ is $T^{1}, \mathrm{SO}(3)$, or $\mathrm{SU}(2)$, and the rank of $H$ must be either 1 or 0 .

4A. Suppose rank $H=1$. Then $G$ is either $\mathrm{SO}(3)$ or $\mathrm{SU}(2)$.

(i) If $G=\mathrm{SO}(3)$, then $H$ is either $\mathrm{SO}(2)$ or $N(\mathrm{SO}(2))$. Since $\mathrm{SO}(3) / N(\mathrm{SO}(2))=R P^{2}$ is not orientable, $H$ should be $\mathrm{SO}(2)$. Since $\mathrm{SO}(3) / \mathrm{SO}(2)$ is $S^{2}$, the orbit space $M^{*}$ is either $S^{2}$ or $D^{2}$. If $M^{*}$ is $S^{2}$, then $M$ is an $S^{2}$-bundle over $S^{2}$. If $M^{*}$ is $D^{2}$, then $\partial D^{2}$ corresponds to the fixed points and int $D^{2}$ corresponds to the principal orbits. Hence $M$ is $S^{4}$. 
(ii) If $G=\mathrm{SU}(2)$, then by an argument similar to that used in (i), $H$ is $\widetilde{\mathrm{SO}}(2)$, and $M$ is either $S^{4}$ or an $S^{2}$-bundle over $S^{2}$.

4B. Suppose rank $H=0$. Then $G$ is $T^{1}, \mathrm{SO}(3)$, or $\mathrm{SU}(2)$.

(i) If $G$ is $T^{1}$, then $H$ must be trivial and $M$ was described in Theorem 2.6.

(ii) If $G=\mathrm{SO}(3)$ and $x^{*}$ and $y^{*}$ are the endpoints of $M^{*}$, then $G_{x}$ and $G_{y}$ are conjugate to $\mathrm{SO}(2), N(\mathrm{SO}(2))$, or $\mathrm{SO}(3)$. By Lemma 2.7, none of $x$ and $y$ are fixed points and $G_{x}$ should be conjugate to $G_{v}$.

(iia) If $G_{x}$ and $G_{v}$ are conjugate to $N(\mathrm{SO}(2))$, then $H$ is a dihedral group $D_{2 n}$ (since $G_{x} / H$ and $G_{y} / H$ must be spheres). Richardson [R] showed that $S^{4}$ admits an action of $\operatorname{SO}(3)$ such that $S^{4} / \operatorname{SO}(3)=\left[x^{*}, y^{*}\right]$, a closed interval, $H=D_{2 n},(\operatorname{SO}(3))(x)=R P^{2}=(\operatorname{SO}(3))(y)$. Since the orbit maps $M \rightarrow M / G$ and $S^{4} \rightarrow S^{4} / \mathrm{SO}(3)$ have cross-sections ([Mo], $[\mathbf{R}]), M$ is equivariantly homeomorphic to $S^{4}$.

(iib) If $G_{x}$ and $G_{v}$ are conjugate to $\mathrm{SO}(2)$, then $H$ should be a cyclic group $Z_{k}$ and $M$ is the space $[0,1] \times \mathrm{SO}(3) / Z_{k}$ with $0 \times \mathrm{SO}(3) / Z_{k}$ collapsed to $\mathrm{SO}(3) / G_{x}\left(\approx S^{2}\right)$ and $1 \times \mathrm{SO}(3) / Z_{h}$ collapsed to $\mathrm{SO}(3) / G_{1}$. $\left(\approx S^{2}\right)$. Let $p$ be the orbit map. Let $A=p^{-1}\left(\left[0, \frac{1}{2}\right]\right)$ and $B=p^{-1}\left(\left[\frac{1}{2}, 1\right]\right)$. From the Mayer-Vietoris sequence for $(M, A, B)$, we have $H_{2}(M ; Q)=$ $Q \oplus Q$ and hence $\chi(M)=4$. Now we consider the action restricted to $G_{\mathrm{r}}$ $\left(\approx T^{\mathrm{l}}\right)$. The set of fixed points under the restricted action is contained in $G(x) \cup G(y)$. Since $N\left(G_{x}\right) / G_{x}$ is $Z_{2}$, there are only two fixed points for $G_{x}$ on $G(x)$, and hence there are at most four fixed points under the restricted action. Let $F\left(G_{x}, M\right)$ denote the set of fixed points. Then it is well known that $\chi\left(F\left(G_{x}, M\right)\right)=\chi(M)=4$. Therefore there are four fixed points for $G_{x}$ on $M$, which implies $G_{x}=G_{v}$. Since $H^{3}(M ; Z)=$ $H_{1}(M ; Z)=0, H_{2}(M ; Z)$ is torsion free and hence $H_{2}(M ; Z)$ is $Z \oplus Z$. The Mayer-Vietoris sequence gives rise to

$$
0 \rightarrow Z \oplus Z \stackrel{i_{*} \oplus j_{*}}{\rightarrow} Z \oplus Z \rightarrow H_{1}\left(\operatorname{SO}(3) / Z_{k} ; Z\right) \rightarrow 0 .
$$

Here $i_{*}$ and $j_{*}$ are induced by inclusions $i: A \rightarrow M$ and $j: B \rightarrow M$ respectively. Since $G_{x}=G_{v}, i$ and $j$ are virtually the same maps. Hence $(Z \oplus Z) / \operatorname{im}\left(i_{*} \oplus j_{*}\right)=Z_{n} \oplus Z_{n}$ for an integer $n$, which contradicts $H_{1}\left(\mathrm{SO}(3) / Z_{k} ; Z\right)=Z_{2 k}$.

(iii) If $G$ is $\operatorname{SU}(2)$ and $\pi$ is the double covering map, then the only subgroups of $\mathrm{SU}(2)$ which do not contain the kernel of $\pi$ are cyclic subgroups of odd order. Hence every subgroup $K$ of $\mathrm{SU}(2)$ contains $Z(\mathrm{SU}(2))$ unless $K$ is a cyclic group of odd order. Since the action was assumed to be effective, $H$ is either $Z_{2 k+1}$ or $e$. By an argument similar to that used in (ii) of $4 \mathrm{~B}$, we can show that $H$ cannot be $Z_{2 k+1}$. If $H$ is the 
identity, then by Lemma 2.7, there are three possibilities:

(a) $x$ and $y$ are fixed points, which implies $M \approx S^{4}$.

(b) $G_{1}$ is conjugate to $\widetilde{\mathrm{SO}}(2)$ and $y$ is a fixed point, which implies $M \approx C P^{2} \# S^{4}$ (Recall: $\mathrm{SU}(2) \rightarrow \mathrm{SU}(2) / \widetilde{\mathrm{SO}}(2)$ is the Hopf bundle).

(c) $G_{、}$ and $G_{v}$ are conjugate to $\widetilde{\mathrm{SO}}(2)$, which implies $M \approx C P^{2} \#-$ $C P^{2}$.

We summarize these in the following table:

TABLE II

\begin{tabular}{|c|c|c|c|c|l|}
\hline $\operatorname{dim} G$ & $\operatorname{rank} H$ & $G$ & $H$ & $M^{*}$ & $M$ \\
\hline 3 & 1 & $\mathrm{SO}(3)$ & $\mathrm{SO}(2)$ & $\begin{array}{l}D^{2} \\
S^{2}\end{array}$ & $\begin{array}{l}S^{4} \\
S^{2} \times S^{2}, C P^{2} \#-C P^{2}\end{array}$ \\
\hline 3 & 1 & $\mathrm{SU}(2)$ & $\widetilde{\mathrm{SO}}(2)$ & $\begin{array}{l}D^{2} \\
S^{2}\end{array}$ & $\begin{array}{l}S^{4} \\
S^{2} \times S^{2}, C P^{2} \#-C P^{2}\end{array}$ \\
\hline 3 & 0 & $\mathrm{SO}(3)$ & $D_{2 n}$ & $D^{1}$ & $S^{4}$ \\
\hline 3 & 0 & $\mathrm{SU}(2)$ & $e$ & $D^{1}$ & $S^{4}, C P^{2}, C P^{2} \#-C P^{2}$ \\
\hline 1 & 0 & $T^{1}$ & $e$ & & Theorem 2.6 \\
\hline
\end{tabular}

5. Conclusion. Suppose a compact connect Lie group $G$ acts on a simply connected 4-manifold $M$. Then it was shown in $\$ 2$ that the rank of $G$ is either 1 or 2 . Let $H$ denote a representative subgroup of the conjugacy class of principal isotropy groups. Then $G, M$, and $H$ are completely determined in $\S \S 3$ and 4 in the cases of rank $G=2$ and rank $G=1$, respectively. Thus we have proved the following.

THEOREM 5.1. If a Lie group $G$, a subgroup $H$, and a manifold $M$ are those denoted above, then $G, H$, and $M$ must be one of the cases represented in Table I (§3) and Table II (§4).

The author would like to thank professors P. E. Conner and Robert Perlis of the Louisiana State University for their encouragement and helpful discussions.

\section{REFERENCES}

[A] J. F. Adams, Lectures on Lie Groups, W. A. Benjamin, (1969).

[B] G. E. Bredon, Introduction to Compact Transformation Groups, Academic Press, (1972).

[E] L. P. Eisenhart, Riemannian Geometry, Princeton University Press, (1949).

$\left[\mathrm{F}_{1}\right] \quad \mathrm{R}$. Fintushel, Circle actions on simply connected 4-manifolds, Trans. Amer. Math. Soc., 230 (1977), 147-171. 
$\left[\mathrm{F}_{2}\right] \quad$ Classification of Circle Actions on 4-Manifolds, Trans. Amer. Math. Soc., 242 (1978), 377-390.

[Ma] L. N. Mann, Gaps in the Dimensions of Compact Transformation Groups, proceedings of the conference on Transformation Groups, Springer-Verlag, (1967), 293-296.

[M-Z] D. Montgomery and L. Zippin, Topological Transformation Groups, Interscience Publishers, (1955).

[Mo] P. S. Mostert, On a compact Lie group acting on a manifold, Annals. of Math., 65 (1957), 447-455; Errata, Annals of Math., 66 (1957), 589, Math. Annalen, 167 (1966), 224.

[O-R] P. Orlik and F. Raymond, Actions of the torus on 4-manifolds I, Trans. Amer. Math. Soc., 152 (1970), 531-559.

[P] J. Pak, Actions of the torus $T^{n}$ on $(n+1)$-manifolds $M^{n+1}$, Pacific J. Math., 44 (1973), 671-674.

[R] R. W. Richardson, Groups acting on the 4-sphere, Illinois J. Math., 5 (1961), 474-485.

[Wa] H. C. Wang, On Finsler spaces with completely integrable equations of killing, J. London Math. Soc., 22 (1947), 5-9.

[Wo] J. A. Wolf, Spaces of constant curvature, Publish or Perish, (1973).

Received May 11, 1981.

Michigan State University

EAST LANSING, MI 48824 



\title{
PACIFIC JOURNAL OF MATHEMATICS EDITORS
}

\author{
Donald BabBitT (Managing Editor) \\ University of California \\ Los Angeles, CA 90024 \\ Hugo RossI \\ University of Utah \\ Salt Lake City, UT 84112 \\ C. C. Moore and Arthur Ogus \\ University of California \\ Berkeley, CA 94720
}

J. DugundiI

Department of Mathematics

University of Southern California

Los Angeles, CA 90089-1113

R. FinN and H. SAMELSON

Stanford University

Stanford, CA 94305

\section{ASSOCIATE EDITORS}
R. ARENS
E. F. BECKENBACH
B. H. NeUmanN
F. WOLF
K. YosHida
(1906-1982)

\section{SUPPORTING INSTITUTIONS}

\author{
UNIVERSITY OF ARIZONA \\ UNIVERSITY OF BRITISH COLUMBIA \\ CALIFORNIA INSTITUTE OF TECHNOLOGY \\ UNIVERSITY OF CALIFORNIA \\ MONTANA STATE UNIVERSITY \\ UNIVERSITY OF NEVADA, RENO \\ NEW MEXICO STATE UNIVERSITY \\ OREGON STATE UNIVERSITY
}

\author{
UNIVERSITY OF OREGON \\ UNIVERSITY OF SOUTHERN CALIFORNIA \\ STANFORD UNIVERSITY \\ UNIVERSITY OF HAWAII \\ UNIVERSITY OF TOKYO \\ UNIVERSITY OF UTAH \\ WASHINGTON STATE UNIVERSITY \\ UNIVERSITY OF WASHINGTON
}

The Supporting Institutions listed above contribute to the cost of publication of this Journal, but they are not owners or publishers and have no responsibility for its content or policies.

Mathematical papers intended for publication in the Pacific Journal of Mathematics should be in typed form or offset-reproduced (not dittoed), double spaced with large margins. Please do not use built up fractions in the text of the manuscript. However, you may use them in the displayed equations. Underline Greek letters in red, German in green, and script in blue. The first paragraph must be capable of being used separately as a synopsis of the entire paper. In particular it should contain no bibliographic references. Please propose a heading for the odd numbered pages of less than 35 characters. Manuscripts, in triplicate, may be sent to any one of the editors. Please classify according to the scheme of Math. Reviews, Index to Vol. 39. Supply name and address of author to whom proofs should be sent. All other communications should be addressed to the managing editor, or Elaine Barth, University of California, Los Angeles, California 90024.

There are page-charges associated with articles appearing in the Pacific Journal of Mathematics. These charges are expected to be paid by the author's University, Government Agency or Company. If the author or authors do not have access to such Institutional support these charges are waived. Single authors will receive 50 free reprints; joint authors will receive a total of 100 free reprints. Additional copies may be obtained at cost in multiples of 50 .

The Pacific Journal of Mathematics is issued monthly as of January 1966. Regular subscription rate: $\$ 132.00$ a year (6 Vol., 12 issues). Special rate: $\$ 66.00$ a year to individual members of supporting institutions.

Subscriptions, orders for numbers issued in the last three calendar years, and changes of address should be sent to Pacific Journal of Mathematics, P.O. Box 969, Carmel Valley, CA 93924, U.S.A. Old back numbers obtainable from Kraus Periodicals Co., Route 100, Millwood, NY 10546.

The Pacific Journal of Mathematics ISSN $0030-8730$ is published monthly by the Pacific Journal of Mathematics at P.O. Box 969, Carmel Valley, CA 93924. Application to mail at Second-class postage rates is pending at Carmel Valley, California, and additional mailing offices. Postmaster: Send address changes to Pacific Journal of Mathematics, P. O. Box 969, Carmel Valley, CA 93924.

PUBLISHED BY PACIFIC JOURNAL OF MATHEMATICS, A NON-PROFIT CORPORATION

Copyright $\odot 1983$ by Pacific Journal of Mathematics 


\section{Pacific Journal of Mathematics}

Vol. 109, No. $2 \quad$ June, 1983

Tibor Bisztriczky, On the singularities of almost-simple plane curves . . . . 257

Peter B. Borwein, On Sylvester's problem and Haar spaces .............. 275

Emilio Bujalance, Cyclic groups of automorphisms of compact nonorientable Klein surfaces without boundary ............... 279

Robert Jay Daverman and John J. Walsh, Acyclic decompositions of

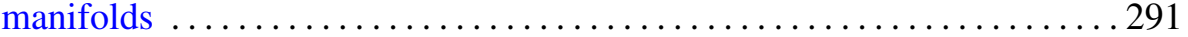

Lester Eli Dubins, Bernstein-like polynomial approximation in higher

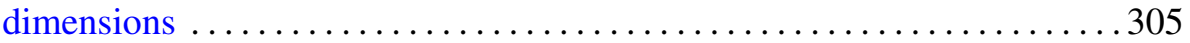

Allan L. Edelson and Jerry Dee Schuur, Nonoscillatory solutions of

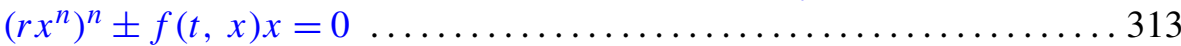

Akira Endô, On units of pure quartic number fields ................. 327

Hector O. Fattorini, A note on fractional derivatives of semigroups and

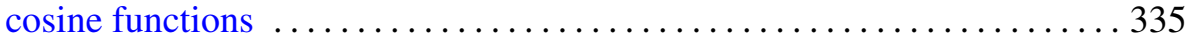

Ronald Fintushel and Peter Sie Pao, Circle actions on homotopy spheres with codimension 4 fixed point set ........................ 349

Stephen Michael Gagola, Jr., Characters vanishing on all but two

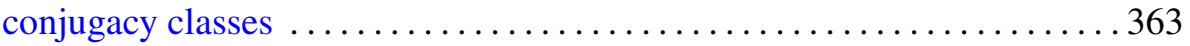

Saverio Giulini, Singular characters and their $L^{p}$ norms on classical Lie

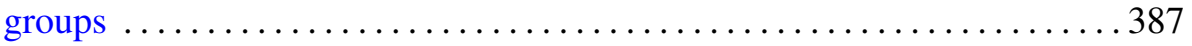

Willy Govaerts, Locally convex spaces of non-Archimedean valued continuous functions

Wu-Chung Hsiang and Bjørn Jahren, A remark on the isotopy classes of diffeomorphisms of lens spaces $\ldots . \ldots \ldots \ldots \ldots \ldots$

Hae Soo Oh, Compact connected Lie groups acting on simply connected 4-manifolds

Frank Okoh and Frank A. Zorzitto, Subsystems of the polynomial system

Knut Øyma, An interpolation theorem for $H_{E}^{\infty}$

Nikolaos S. Papageorgiou, Nonsmooth analysis on partially ordered vector spaces. II. Nonconvex case, Clarke's theory ........ 